Original article

\title{
Glucose transporter 4 mediates LPS-induced IL-6 production in osteoblasts under high glucose conditions
}

\author{
Shunichiro Kato ${ }^{1)}$, Natsuko Tanabe ${ }^{2,3)}$, Mayu Nagao ${ }^{4}$, Jumpei Sekino ${ }^{5}$, Keiko Tomita $^{1)}$, Mayu Sakai ${ }^{1}$, Kimiko Abe ${ }^{3,5)}$, \\ Naoto Suzuki ${ }^{2,3)}$, and Koichiro Ueda ${ }^{3,5)}$ \\ 1) Division of Applied Oral Sciences, Nihon University Graduate School of Dentistry, Tokyo, Japan \\ ${ }^{2)}$ Department of Biochemistry, Nihon University School of Dentistry, Tokyo, Japan \\ ${ }^{3}$ Division of Functional Morphology, Dental Research Center, Nihon University School of Dentistry, Tokyo, Japan \\ ${ }^{4}$ Department of Physiology and Pharmacology, Schulich School of Medicine \& Dentistry, and Bone and Joint Institute, Western University, London, Ontario, Canada \\ ${ }^{5)}$ Department of Dysphagia Rehabilitation, Nihon University School of Dentistry, Tokyo, Japan
}

\begin{abstract}
Purpose: Diabetes causes hyperglycemic disorders due to insufficient activity of insulin, and it also increases blood glucose level. Recent studies have reported the relationship between diabetes and periodontal disease. Periodontitis is advanced by inflammatory cytokines stimulated with LPS. The purpose of this study was to investigate the effects of hyperglycemia on the expression of inflammatory cytokines induced by LPS in osteoblasts. Methods: Cells were cultured for 7 and 14 days in the presence or absence of LPS and glucose. The expression mRNA level of IL-6, RANKL and OCN was determined using real-time PCR. The protein expression of IL-6 and RANKL was also measured using ELISA.

Results: LPS and glucose increased the mRNA expression of IL-6, coupled with a decrease in the mRNA expression of OCN, which is associated with IL-6 and glucose. It also increased the protein expression of IL-6 compared to LPS. However, LPS+Glucose did not affect the mRNA and protein expression of RANKL. Furthermore, GLUT4 inhibitor, WZB117, blocked the stimulatory effect of glucose on LPS-induced IL-6 mRNA expression. WZB117 did not affect LPS-reduced OCN mRNA expression. Conclusion: These results suggest that high glucose levels increase LPSinduced IL-6 expression mediated by GLUT4.
\end{abstract}

Keywords; glucose, GLUT4, LPS, proinflammatory cytokine

\section{Introduction}

The major symptoms of periodontitis, such as gingival inflammation and alveolar bone resorption, are induced by lipopolysaccharide (LPS) [1]. Periodontitis affects nearly 750 million people, which is approximately $10 \%$ of global population worldwide [2]. In previous studies, periodontitis and diabetes showed strong evidence that patients with periodontitis had higher levels of hemoglobin $\mathrm{A} 1 \mathrm{c}$ (HbA1c) compared to healthy subjects $[3,4]$. Patients with poorer control of HbAlc at the baseline have been found to be highly associated with periodontitis in three cohort studies [3]. These epidemiologic studies suggest that periodontitis has an association with the state of hyperglycemia.

LPS is an endotoxin produced by the gram-negative bacteria that bind to Toll-like receptors (TLRs) 4 and 2, inducing inflammatory cytokines or chemokines [5]. Inflammatory cytokine expression induced by LPS affects progression of periodontitis [5]. Interleukin (IL)-1, IL-6, tumor necrosis factor (TNF)- $\alpha$, and receptor activator of nuclear factor kappa B ligand (RANKL) differentiate and activate osteoclasts, which promote bone resorption [6]. Thus, LPS and LPS-induced proinflammatory cytokines are important factors related to the severity of periodontal disease.

Correspondence to Dr. Natsuko Tanabe, Department of Biochemistry, Nihon University School of Dentistry, 1-8-13 Kanda-Surugadai, Chiyoda-ku, Tokyo 101-8310, Japan

Fax:+81332198123 E-mail: tanabe.natsuko@nihon-u.ac.jp

J-STAGE Advance Publication: August 31, 2020

doi.org/10.2334/josnusd.20-0010

DN/JST.JSTAGE/josnusd/20-0010
Previous studies have revealed that glucose can influence the response to LPS $[7,8]$. High glucose concentration $(25 \mathrm{mM})$ increased LPS-induced expression of inducible NO synthase, cyclooxygenase-2, IL-1 $\alpha$, IL- 6 , and TNF- $\alpha$ in macrophage cells [7]. Furthermore, IL-6 and IL-8 expression was induced by LPS in gingival fibroblasts under high glucose conditions in vitro [8]. However, the effects of high glucose levels caused by diabetes on LPS-induced expression of inflammatory cytokines in osteoblasts are poorly understood. Thus, the present study hypothesized that a high concentration of glucose affects LPS-induced inflammatory cytokine levels in osteoblasts. The present study investigated the expression of LPS-induced bone resorption-related proinflammatory cytokines, namely, IL-6, IL-1, and RANKL, as well as determined the effects of glucose transporter 4 (GLUT4) under high glucose levels in the MC3T3-E1 cells. In addition, the expression of osteocalcin (OCN), which affects IL-6 expression in hyperglycemia, was also determined [9].

\section{Materials and Methods}

\section{Cell culture}

MC3T3-E1 are mouse osteoblastic cell lines purchased from Riken Bio Resource Center (Tsukuba, Japan). Cells were cultured in the manner of the previous study [10]. Cells were stimulated with $100 \mathrm{ng} / \mathrm{mL}$ LPS derived from Escherichia coli (Sigma-Aldrich, St. Louis, MO, USA), $1 \mathrm{mg} / \mathrm{mL}$ bovine serum albumin (BSA, Fujifilm Wako Pure Chemical Corp., Osaka, Japan), and/or $22 \mathrm{mM}$ glucose (Fujifilm Wako Pure Chemical Corp.) or left untreated. Cells were treated with or without WZB117 $(1.0 \mu \mathrm{M}$, Tocris, Bristol, UK), a GLUT4 inhibitor, in condition medium.

\section{Real-time polymerase chain reaction (real-time PCR)}

MC3T3-E1 cells were collected in each condition on days 7 and 14 of culture as a sample. mRNA was isolated from sample and has been reverse transcription to complementary DNA. The target mRNA level was measured using real-time PCR in the manner of the previous study [10]. PCR primer sequences used in real-time PCR are described in Table 1. Target mRNA level was calculated, which was normalized to the mRNA level of beta-actin ( $\beta$-actin) was used as an internal control.

\section{Enzyme-linked immunosorbent assay (ELISA)}

The concentrations of IL-6 and RANKL in the cell culture supernatant at 14 days were quantified using ELISA kits (Quantikine; R\&D Systems, Minneapolis, MN, USA) in the manner of the previous study [10].

\section{Statistical analysis}

Data represented the results of three independent experiments. Primary data were confirmed by Shapiro-Wilk test and homoscedasticity by Bartlett test. Differences between groups were analyzed by one-way analysis of variance (ANOVA), and differences were considered statistically significant at $P<0.05$. Statistical analysis was performed with EZR software (EZR 1.23; Jichi Medical University Saitama Medical Center, Saitama, Japan) [11]. Each value expressed the mean \pm standard division (SD). 
Table 1 PCR primers used in the experiments

\begin{tabular}{lll}
\hline Target & Primers & GenBank Acc. \\
\hline IL-6 & 5'-CAACGATGATGCACTTGCAGA-3' & NM_031168.1 \\
& 5'-CTCCAGGTAGCTATGGTACTCCAGA-3' & \\
RANKL & 5'-CATGTGCCACTGAGAACCTTGAA-3' & NM_011613.3 \\
& 5'-CAGGTCCCAGCGCAATGTAAC-3' & \\
OCN & 5'-AAGCAGGAGGGCAATAAGGT-3' & NM_007541.2 \\
& 5'-ACCCTGCTACGACAACGTGCC-3' & \\
ß-actin & 5'-CATCCGTAAAGACCTCTATGCCAAC-3' & NM_007393.5 \\
& 5'-ATGGAGCCACCGATCCACA-3' & \\
\hline
\end{tabular}
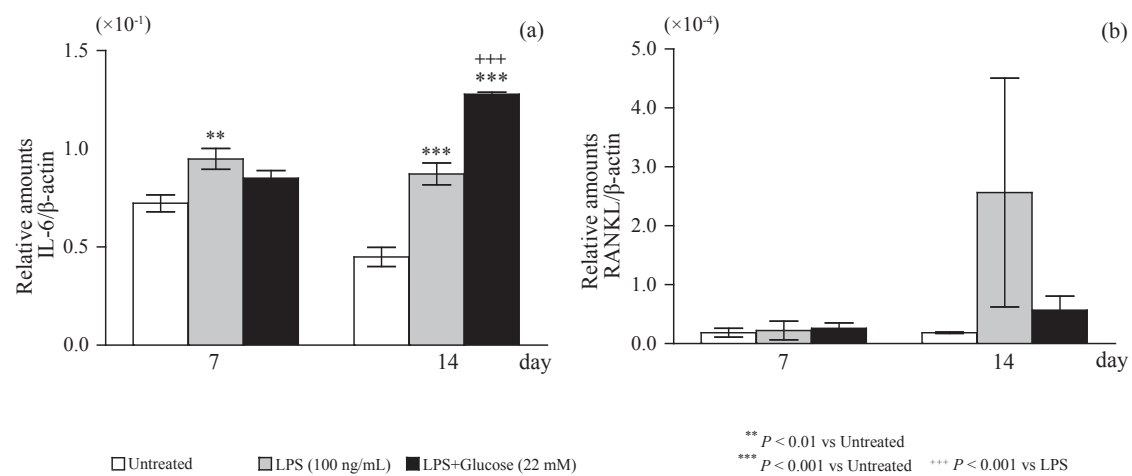

(b)

口Untreated $\quad \square$ LPS $(100 \mathrm{ng} / \mathrm{mL}) \quad$ L LPS + Glucose $(22 \mathrm{mM})$

Fig. 1 Cells were stimulated with glucose $(22 \mathrm{mM})$ and/or LPS $(100 \mathrm{ng} / \mathrm{mL})$ or left unstimulated (untreated control), and the gene expression of IL-6 (a) and RANKL (b) was determined on days 7 and 14 of culture using real-time PCR. ${ }^{*} P<0.05$, vs. untreated control, ${ }^{+} P<0.05$, vs. LPS

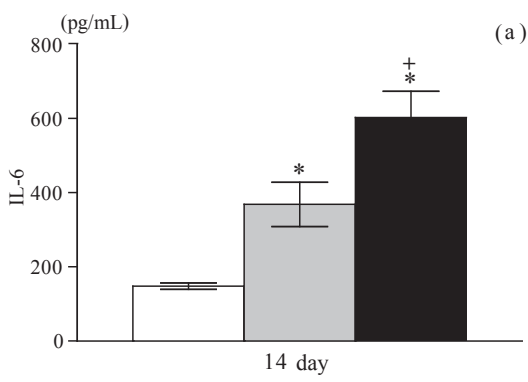

口Untreated (a)

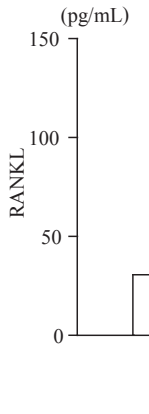

(b)

Fig. 2 Cells were stimulated with glucose $(22 \mathrm{mM})$ and/or LPS $(100 \mathrm{ng} / \mathrm{mL})$ or left without stimulation (untreated control) and the protein expression of IL-6 (a) and RANKL (b) was determined on day 14 of culture using ELISA. ${ }^{*} P<0.05$ vs. untreated control, ${ }^{+} P<0.05$, vs LPS
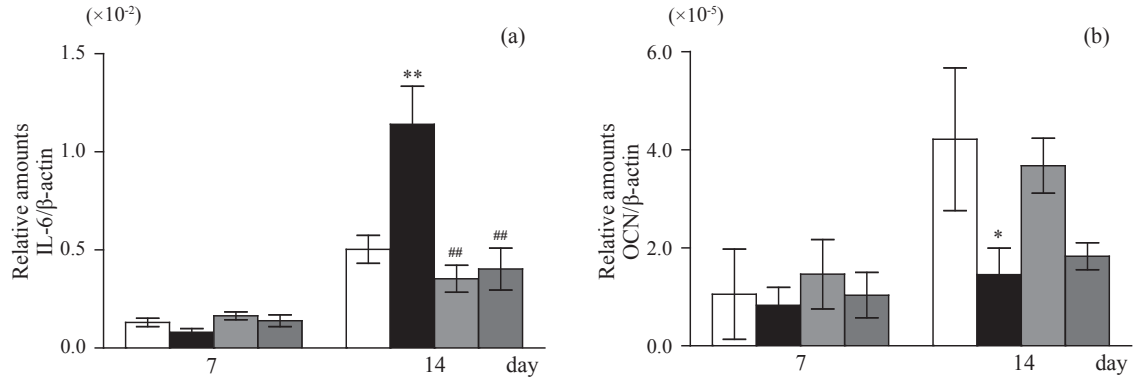

口Untreated

LPS+Glucose $(22 \mathrm{mM})$

$\square$ Untreated+WZB1 $17(1.0 \mu \mathrm{M})$

$\square$ LPS+Glucose+WZB117

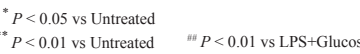

Fig. 3 Cells were stimulated with glucose $(22 \mathrm{mM})$, and $/$ or LPS $(100 \mathrm{ng} / \mathrm{mL})$ in the presence or absence of GLUT4 inhibitor WZB117 $(1.0 \mu \mathrm{M})$ or left without stimulation (untreated control) and the gene expression of IL-6 (a) and OCN (b) was determined on days 7 and 14 of culture using real-time PCR. ${ }^{*} P<0.05$, vs. untreated control, ${ }^{\#} P<0.05$, vs LPS+Glucose

\section{Results}

The Shapiro-Wilk and Bartlett tests run on the results of the Figs.1, 2, and 3 showed $P$ values greater than 0.05 . Thus, the results of Figs.1, 2, and 3 were analyzed by one-way ANOVA and Tukey's multiple comparisons.

Effects of glucose on IL-6 and RANKL mRNA level induced by LPS LPS and LPS+Glucose significantly increased the mRNA level of IL-6 on days 7 and/or 14 of culture (by 1.31-, 1.90, and 2.80-fold, respectively) compared to that of the untreated control $(P=0.0057: 7$ day, $P=$ $0.0000012: 14$ day LPS, $P=0.000057: 14$ day LPS+Glucose). Additionally, LPS+Glucose significantly increased the mRNA expression of IL-6 compared to that of LPS alone on days 14 of culture (by 1.47 -fold, $P=$ 0.000071) (Fig. 1a). However, LPS and LPS+Glucose did not affect the mRNA expression of RANKL (Fig. 1b). 
Effects of glucose on IL-6 and RANKL protein level induced by LPS LPS and LPS+Glucose significantly increased the protein expression of IL-6 (by 2.58- and 4.17-fold, respectively) on day 14 of culture compared to that of the untreated control $(P=0.0060$ : LPS, $P=0.000121$ : LPS+Glucose) (Fig. 2a). LPS+Glucose also significantly increased the protein expression of IL-6 compared to that of LPS alone (by 1.62-fold, $P=0.0044$ ) (Fig. 2a). Moreover, LPS significantly enhanced the protein expression of RANKL on day 14 of culture compared to that of the untreated control (by 3.46fold, $P=0.033)$. However, glucose did not affect the protein expression of RANKL (Fig. 2b).

\section{Effects of GLUT4 antagonist on the mRNA level of IL-6 and OCN}

To elucidate the mechanism through which glucose increases LPS-induced IL-6 expression, the present study determined the effect of GLUT4 inhibitor WZB117 on the mRNA expression of IL-6. LPS+Glucose induced the mRNA expression of IL-6 compared to that of the untreated control (by 2.27 -fold, $P=0.0052$ ) on day 14 of culture. In contrast, WZB117 inhibited the stimulatory effects of LPS+Glucose on the mRNA expression of IL-6 (by 0.36 -fold, $P=0.0021$ ) (Fig. 3a). LPS+Glucose reduced the mRNA expression of OCN on day 14 of culture compared to that of the untreated control (by 0.35 -fold, $P=0.043$ ). WZB117 also did not affect LPS-reduced OCN mRNA expression (Fig. 3b).

\section{Discussion}

LPS induces the secretion of proinflammatory factors, namely, TNF- $\alpha$, IL-1, IL-6, and RANKL, and advanced periodontitis [1,9,12]. Proinflammatory cytokines directly affect the periodontal ligament. However, previous studies have shown that these cytokines also stimulate alveolar bone resorption, which involves osteoblasts and osteoclasts [1]. In addition, some previous studies have indicated that IL- 6 and C-reactive protein are correlated with type 2 diabetes [13]. Based on these findings, this present study hypothesized that high glucose levels affect the expression of proinflammatory cytokines in osteoblasts. Thus, the purpose of this study was to investigate the effects of glucose on the expression of proinflammatory cytokines induced by LPS in osteoblasts.

Many cell types in inflammatory bone disease secrete IL-6. Previous studies have reported the inhibition of osteoarthritis development in IL-6 knockout mice [14-16]. These findings indicate that IL-6 is a major factor involved in rheumatoid arthritis and plays a direct role in the bone [17]. In contrast, IL- 6 also binds to its soluble receptor, activating osteoblast differentiation in vitro $[18,19]$. However, IL-6 does not affect osteoblast proliferation [20].

RANKL, a member of the TNF superfamily, is a transmembrane ligand expressed by osteoblasts [21,22]. RANKL strongly induces osteoclast differentiation and survival. It is also expressed on stromal, B-lymphoid lineage, and activated T-cells [21]. Furthermore, soluble RANKL is secreted by various cells [21-23]. Receptor activator of nuclear factor kappa B in the monocyte-macrophage lineage cells bind to RANKL and differentiate from precursor cells to mature osteoclasts that have the ability of bone resorption [24]. RANKL knockout mice exhibit a complete lack of osteoclasts, which causes severe osteopetrosis [25]. In contrast, RANKL transgenic mice reduce bone mass by increasing the number of osteoclasts [26]. These studies indicate that IL-6 and RANKL are major factors for bone resorption-implicated inflammation in osteoarthritis and periodontitis. The current study showed that glucose increased the LPS-induced expression of IL-6, whereas glucose did not affect LPS-induced RANKL expression (Figs. 1 and 2). However, previous studies have revealed that IL-6 induces the production of RANKL, associated with indirect osteoclastogenesis [27]. Thus, the results of this study suggest that LPS+Glucose-induced expression of IL-6 might stimulate osteoclastogenesis.

A family of GLUT isoforms mediates cellular glucose uptake. These isoforms have the following three high-affinity transporters: GLUT1, which is expressed in various tissues including tumors; GLUT3, which is expressed particularly in brains (neurons); and GLUT4, which is expressed in insulin-sensitive tissues such as striated muscle and adipose tissue [28]. GLUT1, GLUT3, and GLUT4 are expressed in osteoblasts in vitro. However, GLUT1 and GLUT3 are constantly expressed during the course of osteoblast differentiation [29]. GLUT4 expression is implicated in the enhancement of insulin-stimulated glucose uptake [29]. Downregu- lation of GLUT4 expression eliminates insulin-stimulated glucose uptake in osteoblasts in vitro and reduces osteoblast proliferation and differentiation. Furthermore, GLUT4 is expressed in osteoblasts, osteocytes, and chondrocytes, similar to that of the adjacent skeletal muscles, in vivo [29]. Next, this study focused on the effect of GLUT4 on the expression of IL-6 induced by the stimulation of LPS under high glucose concentrations in the present study. GLUT4 inhibitor WZB117 blocked the stimulatory effects of LPS and glucose on IL-6 mRNA expression on day 14 of culture (Fig. 3a). These results showed that glucose enhanced LPS-induced expression of IL-6 through GLUT4 in osteoblasts.

A previous study has reported that the plasma concentration of IL- 6 and glucose increases after orthopedic surgery, whereas the plasma concentration of OCN decreases [30]. In a previous study, 108 patients with type 2 diabetes exhibited increased plasma IL-6 levels and decreased plasma OCN levels compared to those for non-diabetic controls [9]. These studies revealed that inflammation might be correlated with hyperglycemia and OCN expression $[9,30]$. The plantaris and soleus muscle injection of IL-6 induces GLUT4 expression in mice in vivo [31]. IL-6 also enhances GLUT4 expression in $\mathrm{C} 2 \mathrm{C} 12$ cells that differentiate in vitro. In contrast, OCN inhibits TNF- $\alpha$, IL- 6 , and IL- $1 \beta$ expression and increases GLUT4 expression in adipocytes [32]. TNF- $\alpha$ stimulation also enhances IL-6 mRNA expression and lowers OCN mRNA expression in osteoblasts. However, the expression of GLUT4 does not affect OCN in bone tissue in vivo and in vitro [32]. These conflicting observations showed the expression of GLUT4 independently in each tissue. Additionally, that the expression of GLUT4 may also cause a difference in the physiological activation is a conceivable possibility. The present study showed that stimulation of LPS under high glucose levels reduced OCN mRNA expression. GLUT4 inhibitor did not affect the mRNA expression of OCN in osteoblasts stimulated with LPS under high glucose levels (Fig. 3b). These results showed that GLUT4 was not associated with a decrease in OCN expression by glucose and LPS. Thus, in future studies, it will be necessary to investigate the relationship between insulin effects and $\mathrm{OCN}$ expression to elucidate the mechanism underlying inflammation and hyperglycemia in osteoblasts.

In summary, high glucose levels increased LPS-induced IL- 6 and IL-1 $\alpha$ expression mediated by GLUT4. However, GLUT4 did not decrease OCN expression in osteoblasts. Glucose did not exert a stimulatory effect on the LPS-induced protein expression of RANKL. These phenomena might be related to the mechanism underlying the relationship between periodontitis and type 2 diabetes in vitro. To the best of this knowledge, this is the first study to show that GLUT4 mediates IL-6 expression under high glucose levels in osteoblasts.

\section{Acknowledgments}

This study was supported by JSPS KAKENHI Grant Number 18K09609, a grant from the Sato fund and Uemura fund, Nihon University School of Dentistry, a grant from the Dental Research Center, Nihon University and Nihon University Multidisciplinary Research Grant for 2017 (017-019).

\section{Conflict of interest}

There is no conflict of interest to declare.

\section{References}

1. Page RC (1991) The role of inflammatory mediators in the pathogenesis of periodontal disease. J Periodontal Res 26, 230-242.

2. Kassebaum NJ, Bernabé E, Dahiya M, Bhandari B, Murray CJL, Marcenes W (2014) Global burden of severe periodontitis in 1990-2010: a systematic review and meta- regression. J Dent Res 93, 1045-1053.

3. Sanz M, Ceriello A, Buysschaert M, Chapple I, Demmer RT, Graziani F et al. (2018) Scientific evidence on the links between periodontal diseases and diabetes: consensus report and guidelines of the joint workshop on periodontal diseases and diabetes by the International Diabetes Federation and the European Federation of Periodontology. Diabetes Res Clin Pract 137, 231-241.

4. Graziani F, Gennai S, Solini A, Petrini M (2018) A systematic review and meta-analysis of epidemiologic observational evidence on the effect of periodontitis on diabetes an update of the EFP-AAP review. J Clin Periodontol 45, 167-187.

5. Rhee SH (2014) Lipopolysaccharide: basic biochemistry, intracellular signaling, and physiological impacts in the gut. Intest Res 12, 90-95.

6. Liu J, Wang S, Zhang P, Said-Al-Naief N, Michalek SM, Feng X (2009) Molecular mechanism of the bifunctional role of lipopolysaccharide in osteoclastogenesis. J Biol Chem 284, 12512-12523.

7. Hwang JS, Kwon MY, Kim KH, Lee Y, Lyoo IK, Kim JE et al. (2017) Lipopolysaccharide (LPS)-stimulated iNOS induction is increased by glucosamine under normal glucose conditions but is inhibited by glucosamine under high glucose conditions in macrophage cells. 
J Biol Chem 292, 1724-1736.

8. Chiu HC, Fu MM, Yang TS, Fu E, Chiang CY, Tu HP et al. (2017) Effect of high glucose, Porphyromonas gingivalis lipopolysaccharide and advanced glycation end-products on production of interleukin-6/-8 by gingival fibroblasts. J Periodontal Res 52, 268-276.

9. Sarkar PD, Choudhury AB (2013) Relationships between serum osteocalcin levels versus blood glucose, insulin resistance and markers of systemic inflammation in central Indian type 2 diabetic patients. Eur Rev Med Pharmacol Sci 17, 1631-1635.

10. Shionome C, Kawato T, Tanabe N, Kariya T, Sanuki R, Koyama Y et al. (2012) Compressive force induces the expression of bone remodeling-related proteins via interleukin-11 production in MC3T3-E1 cells. J Hard Tissue Biol 21, 65-74.

11. Kanda Y (2013) Investigation of the freely available easy-to-use software 'EZR' for medical statistics. Bone Marrow Transplant 48, 452-458.

12. Shoji M, Tanabe N, Mitsui N, Tanaka H, Suzuki N, Takeichi O et al. (2006) Lipopolysaccharide stimulates the production of prostaglandin E2 and the receptor Ep4 in osteoblasts. Life Sci 78, 2012-2018

13. Wang X, Bao W, Liu J, Ouyang YY, Wang D, Rong S et al. (2013) Inflammatory markers and risk of type 2 diabetes: a systematic review and meta-analysis. Diabetes Care 36, 166175 .

14. Ohshima S, Saeki Y, Mima T, Sasai M, Nishioka K, Nomura S et al. (1998) Interleukin 6 plays a key role in the development of antigen-induced arthritis. Proc Natl Acad Sci U S A 95, 8222-8226.

15. Sasai M, Saeki Y, Ohshima S, Nishioka K, Mima T, Tanaka T et al. (1999) Delayed onset and reduced severity of collagen-induced arthritis in interleukin-6-deficient mice. Arthritis Rheum 42, 1635-1643.

16. Boe A, Baiocchi M, Carbonatto M, Papoian R, Serlupi-Crescenzi O (1999) Interleukin 6 knock-out mice are resistant to antigen-induced experimental arthritis. Cytokine 11, 1057 1064.

17. Le Goff B, Blanchard F, Berthelot JM, Heymann D, Maugars Y (2010) Role for interleukin-6 in structural joint damage and systemic bone loss in rheumatoid arthritis. Joint Bone Spine 77, 201-205.

18. Bellido T, Stahl N, Farruggella TJ, Borba V, Yancopoulos GD, Manolagas SC (1996) Detection of receptors for interleukin-6, interleukin-11, leukemia inhibitory factor, oncostatin M, and ciliary neurotrophic factor in bone marrow stromal/ osteoblastic cells. J Clin Invest 97, 431-437.

19. Taguchi Y, Yamamoto M, Yamate T, Lin SC, Mocharla H, De Togni P et al. (1998) Interleukin-6-type cytokines stimulate mesenchymal progenitor differentiation toward the osteoblastic lineage. Proc Assoc Am Physicians 110, 559-574.

20. Heymann D, Rousselle AV (2000) gp130 cytokine family and bone cells. Cytokine 12,
$1455-1468$

21. Lacey DL, Timms E, Tan HL, Kelley MJ, Dunstan CR, Burgess T et al. (1998) Osteoprotegerin ligand is a cytokine that regulates osteoclast differentiation and activation. Cell 93 $165-176$

22. Manabe N, Kawaguchi H, Chikuda H, Miyaura C, Inada M, Nagai R et al. (2001) Connection between B lymphocyte and osteoclast differentiation pathways. J Immunol 167 , 2625-2631.

23. Yasuda H, Shima N, Nakagawa N, Yamaguchi K, Kinosaki M, Mochizuki S et al. (1998) Osteoclast differentiation factor is a ligand for osteoprotegerin/osteoclastogenesis-inhibitory factor and is identical to TRANCE/RANKL. Proc Natl Acad Sci U S A 95, 3597-3602.

24. Takayanagi H (2007) Osteoimmunology: shared mechanisms and crosstalk between the immune and bone systems. Nat Rev Immunol 7, 292-304.

25. Kong YY, Yoshida H, Sarosi I, Tan HL, Timms E, Capparelli C et al. (1999) OPGL is a key regulator of osteoclastogenesis, lymphocyte development and lymph-node organogenesis. Nature 397, 315-323.

26. Mizuno A, Kanno T, Hoshi M, Shibata O, Yano K, Fujise N et al. (2002) Transgenic mice overexpressing soluble osteoclast differentiation factor (sODF) exhibit severe osteoporosis. J Bone Miner Metab 20, 337-344.

27. Palmqvist P, Persson E, Conaway HH, Lerner UH (2002) IL-6, leukemia inhibitory factor, and oncostatin $\mathrm{M}$ stimulate bone resorption and regulate the expression of receptor activator of NF-kB ligand, osteoprotegerin, and receptor activator of NF- $\mathrm{kB}$ in mouse calvariae. J Immunol 169, 3353-3362.

28. Zoidis E, Ghirlanda-Keller C, Schmid C (2011) Stimulation of glucose transport in osteoblastic cells by parathyroid hormone and insulin-like growth factor I. Mol Cell Biochem $348,33-42$.

29. Li Z, Frey JL, Wong GW, Faugere MC, Wolfgang MJ, Kim JK et al. (2016) Glucose transporter-4 facilitates insulin-stimulated glucose uptake in osteoblasts. Endocrinology 157 , 4094-4103.

30. Nicholson G, Bryant AE, Macdonald IA, Hall GM (2002) Osteocalcin and the hormonal, inflammatory and metabolic response to major orthopaedic surgery. Anaesthesia 57, 319325.

31. Ikeda S, Tamura Y, Kakehi S, Sanada H, Kawamori R, Watada H (2016) Exercise-induced increase in IL-6 level enhances GLUT4 expression and insulin sensitivity in mouse skeletal muscle. Biochem Biophys Res Commun 473, 947-952.

32. Guedes JAC, Esteves JV, Morais MR, Zorn TM, Furuya DT (2018) Osteocalcin improves insulin resistance and inflammation in obese mice: participation of white adipose tissue and bone. Bone $115,68-82$ 